\title{
Oscillation of third-order delay difference equations with negative damping term
}

\begin{abstract}
The aim of this paper is to investigate the oscillatory and asymptotic behavior of solutions of a third-order delay difference equation. By using comparison theorems, we deduce oscillation of the difference equation from its relation to certain associated first-order delay difference equations or inequalities. Examples are given to illustrate the main results.
\end{abstract}

1. Introduction. In this paper, we study the oscillatory behavior of solutions of the third-order delay difference equation of the form

$$
\Delta^{3} y_{n}-p_{n} \Delta y_{n+1}+q_{n} f\left(y_{n-\ell}\right)=0, \quad n \geq n_{0}
$$

where $\left\{p_{n}\right\}$ and $\left\{q_{n}\right\}$ are real sequences, $n_{0} \in \mathbb{N}_{0}$, and $f$ is a real-valued continuous function. Throughout this paper, we assume the following conditions without further mention:

$\left(\mathrm{H}_{1}\right)\left\{p_{n}\right\}$ is a nonnegative real sequence and $\left\{q_{n}\right\}$ is a positive real sequence for all $n \geq n_{0}$;

$\left(\mathrm{H}_{2}\right) \ell \in \mathbb{N}$

$\left(\mathrm{H}_{3}\right) u f(u)>0, f$ is nondecreasing for $u \neq 0$, and

$$
-f(u v) \geq f(u v) \geq f(u) f(v) \text { for } u v>0 .
$$

2010 Mathematics Subject Classification. 39A10.

Key words and phrases. Third-order delay difference equation, comparison theorems, oscillation, asymptotic behavior. 
By a solution of (1.1), we mean a nontrivial real sequence $\left\{y_{n}\right\}$, defined for all $n \geq n_{0}-\ell$, and satisfying (1.1) for all $n \geq n_{0}$. A nontrivial solution $\left\{y_{n}\right\}$ of (1.1) is said to be oscillatory if it is neither eventually positive nor eventually negative, and nonoscillatory otherwise. Any difference equation is called nonoscillatory (oscillatory) if all its solutions are nonoscillatory (oscillatory).

In recent years, there is a great interest in studying the oscillatory and asymptotic behavior of solutions of several classes of third-order functional difference equations, see [3]-[8], [10]-[13], [15]-[17] and the references cited therein. In [5], [10], [12], [13], the authors considered the difference equation

$$
\Delta\left(a_{n} \Delta\left(b_{n} \Delta y_{n}\right)\right)+p_{n} \Delta y_{n+1}+q_{n} f\left(y_{n-\ell}\right)=0, \quad n \geq n_{0},
$$

and established some new sufficient conditions for the oscillation and asymptotic behavior of solutions of (1.2). Very recently, in [6], the authors discussed the oscillatory and asymptotic behavior of solutions of the equation

$$
\Delta\left(a_{n} \Delta\left(b_{n}\left(\Delta y_{n}\right)^{\alpha}\right)\right)+p_{n}\left(\Delta y_{n+1}\right)^{\alpha}+q_{n} f\left(y_{n-\ell}\right)=0, \quad n \geq n_{0} .
$$

In (1.2) and (1.3), the authors assumed the coefficient sequence of the damping term is positive, and therefore, in this paper, we investigate the oscillatory and asymptotic behavior of solutions of (1.1) by assuming the coefficient of the damping term is negative. Thus the results obtained in this paper are new and complement those in [5], [6], [10], [12], [13].

In Section 2, we present some preliminary results, and in Section 3, we obtain some sufficient conditions for the oscillation of all solutions of (1.1). Examples are provided in Section 4 in order to illustrate the importance of the main results.

2. Preliminary Results. In this section, we present some preliminary results, which are used to prove our main theorems. The main theorems, presented in Section 3, relate the properties of solutions of third-order delay difference equations of the form (1.1) to those of solutions of an auxiliary second-order linear difference equation of the form

$$
\Delta^{2} z_{n}-p_{n} z_{n+1}=0, \quad n \geq n_{0} .
$$

The first result is based on an equivalent representation for the linear difference operator

$$
L\left(y_{n}\right):=\Delta^{3} y_{n}-p_{n} \Delta y_{n+1}
$$

in terms of a positive solution $\left\{z_{n}\right\}$ of (2.1). We prove that under the assumption $\left(\mathrm{H}_{1}\right),(2.1)$ is nonoscillatory.

Lemma 2.1. Let $\left\{p_{n}\right\}$ be a nonnegative real sequence for all $n \geq n_{0}$. Then (2.1) is nonoscillatory. 
Proof. Equation (2.1) can be written as

$$
z_{n+2}+z_{n}=\left(p_{n}+2\right) z_{n+1}, \quad n \geq n_{0},
$$

and we have

$$
\frac{1}{\left(p_{n}+2\right)\left(p_{n+1}+2\right)} \leq \frac{1}{4}, \quad n \geq n_{0} .
$$

Hence by applying [2, Theorem 1.8.9], we see that (2.1) is nonoscillatory.

Lemma 2.2. Assume that (2.1) has a positive solution $\left\{z_{n}\right\}$. Then the operator (2.2) can be represented as

$$
L\left(y_{n}\right)=\frac{\Delta\left(z_{n} z_{n+1} \Delta\left(\frac{\Delta y_{n}}{z_{n}}\right)\right)}{z_{n+1}} .
$$

Proof. It is easy to see that

$$
\begin{aligned}
L\left(y_{n}\right) & =\Delta^{3} y_{n}-p_{n} \Delta y_{n+1} \\
& =\frac{z_{n+1} \Delta^{3} y_{n}+\left(\Delta z_{n}\right)\left(\Delta^{2} y_{n}\right)-\left(\Delta z_{n}\right)\left(\Delta^{2} y_{n}\right)-\left(\Delta^{2} z_{n}\right)\left(\Delta y_{n+1}\right)}{z_{n+1}} \\
& =\frac{\Delta\left(z_{n} \Delta^{2} y_{n}-\left(\Delta z_{n}\right)\left(\Delta y_{n}\right)\right)}{z_{n+1}} \\
& =\frac{\Delta\left(z_{n} z_{n+1} \Delta\left(\frac{\Delta y_{n}}{z_{n}}\right)\right)}{z_{n+1}},
\end{aligned}
$$

completing the proof.

Lemma 2.3. If $\left\{z_{n}\right\}$ is a positive solution of (2.1), then (1.1) can be written in the form

$$
\Delta\left(z_{n} z_{n+1} \Delta\left(\frac{\Delta y_{n}}{z_{n}}\right)\right)+q_{n} z_{n+1} f\left(y_{n-\ell}\right)=0 .
$$

Proof. The proof follows from Lemma 2.2.

For our discussion, it is convenient to have (2.3) in canonical form, that is, we require in the sequel that

$$
\sum_{n=n_{0}}^{\infty} z_{n}=\infty
$$

and

$$
\sum_{n=n_{0}}^{\infty} \frac{1}{z_{n} z_{n+1}}=\infty
$$

To prove our main results, we find conditions that guarantee the existence of positive solutions of (2.1). The following result is a special case of the discrete Kneser theorem (see [1]) dealing with the structure of nonoscillatory solutions of (2.1). 
Lemma 2.4. Under the assumption $\left(H_{1}\right),(2.1)$ has a principal (recessive) solution $\left\{u_{n}\right\}$ satisfying

$$
u_{n}>0, \quad \Delta u_{n}<0, \quad \Delta^{2} u_{n} \geq 0, \quad n \geq n_{0}
$$

and a nonprincipal (dominant) solution $\left\{v_{n}\right\}$ satisfying

$$
v_{n}>0, \quad \Delta v_{n}>0, \quad \Delta^{2} v_{n} \geq 0, \quad n \geq n_{0} .
$$

Next, we study the behavior of solutions of (1.1) with the help of its equivalent representation (2.3). In view of well-known results in [1], [2], we have the following structure of nonoscillatory solutions of (1.1).

Lemma 2.5. Let $\left\{z_{n}\right\}$ be a positive solution of (2.1) satisfying (2.5). Then, every positive solution $\left\{y_{n}\right\}$ of (1.1) is inside one of the following two classes:

(I) $y_{n}>0, \Delta y_{n}>0, \Delta\left(\frac{\Delta y_{n}}{z_{n}}\right)>0, \Delta\left(z_{n} z_{n+1} \Delta\left(\frac{\Delta y_{n}}{z_{n}}\right)\right)<0$;

(II) $y_{n}>0, \Delta y_{n}<0, \Delta\left(\frac{\Delta y_{n}}{z_{n}}\right)>0, \Delta\left(z_{n} z_{n+1} \Delta\left(\frac{\Delta y_{n}}{z_{n}}\right)\right)<0$.

We conclude this section with the following notation. We say that (1.1) has Property (B) provided all its nonoscillatory solutions $\left\{y_{n}\right\}$ satisfy the condition

$$
y_{n} \Delta y_{n}<0
$$

3. Oscillation Results. In the sequel, it is tacitly assumed that the sequence $\left\{z_{n}\right\}$ is a solution of (2.1) and satisfies (2.5). We begin with the following theorem.

Theorem 3.1. Let $\left\{z_{n}\right\}$ be a positive solution of (2.1) such that (2.4) and (2.5) hold. If the first-order delay difference equation

$$
\Delta x_{n}+q_{n} z_{n+1} f\left(\sum_{s=n_{1}}^{n-\ell-1} z_{s} \sum_{t=n_{1}}^{s-1} \frac{1}{z_{t} z_{t+1}}\right) f\left(x_{n-\ell}\right)=0, \quad n \geq n_{1}
$$

is oscillatory, then (1.1) has Property (B).

Proof. Let $\left\{y_{n}\right\}$ be a positive solution of (1.1) for all $n \geq n_{1}$, where $n_{1} \geq n_{0}$ is some integer. It follows from Lemma 2.5 that $\left\{y_{n}\right\}$ belongs to either class (I) or class (II) for all $n \geq n_{1}$. We shall prove that $\left\{y_{n}\right\}$ belongs to class (II). For this, we assume that $\left\{y_{n}\right\}$ belongs to class (I). Define a sequence $\left\{x_{n}\right\}$ by

$$
x_{n}=z_{n} z_{n+1} \Delta\left(\frac{1}{z_{n}} \Delta y_{n}\right), \quad n \geq n_{1} .
$$


Then $\left\{x_{n}\right\}$ is strictly decreasing, and we conclude

$$
\begin{aligned}
\frac{\Delta y_{n}}{z_{n}} & \geq \sum_{s=n_{1}}^{n-1} \Delta\left(\frac{\Delta y_{s}}{z_{s}}\right)=\sum_{s=n_{1}}^{n-1} \frac{1}{z_{s} z_{s+1}}\left[z_{s} z_{s+1} \Delta\left(\frac{\Delta y_{s}}{z_{s}}\right)\right] \\
& \geq x_{n} \sum_{s=n_{1}}^{n-1} \frac{1}{z_{s} z_{s+1}}
\end{aligned}
$$

i.e.,

$$
\Delta y_{n} \geq x_{n} z_{n} \sum_{s=n_{1}}^{n-1} \frac{1}{z_{s} z_{s+1}} .
$$

Summing (3.2) from $n_{1}$ to $n-1$ yields

$$
y_{n} \geq \sum_{s=n_{1}}^{n-1} x_{s} z_{s} \sum_{t=n_{1}}^{s-1} \frac{1}{z_{t} z_{t+1}} \geq x_{n} \sum_{s=n_{1}}^{n-1} z_{s} \sum_{t=n_{1}}^{s-1} \frac{1}{z_{t} z_{t+1}} .
$$

Hence

$$
y_{n-\ell} \geq x_{n-\ell} \sum_{s=n_{1}}^{n-\ell-1} z_{s} \sum_{t=n_{1}}^{s-1} \frac{1}{z_{t} z_{t+1}} .
$$

Combining (3.3) with (2.3) and using $\left(\mathrm{H}_{3}\right)$, we obtain

$$
-\Delta x_{n}=z_{n+1} q_{n} f\left(y_{n-\ell}\right) \geq z_{n+1} q_{n} f\left(\sum_{s=n_{1}}^{n-\ell-1} z_{s} \sum_{t=n_{1}}^{s-1} \frac{1}{z_{t} z_{t+1}}\right) f\left(x_{n-\ell}\right) .
$$

Therefore, $\left\{x_{n}\right\}$ is a positive solution of the delay difference inequality

$$
\Delta x_{n}+z_{n+1} q_{n} f\left(\sum_{s=n_{1}}^{n-\ell-1} z_{s} \sum_{t=n_{1}}^{s-1} \frac{1}{z_{t} z_{t+1}}\right) f\left(x_{n-\ell}\right) \leq 0 .
$$

It follows from [17, Lemma 2.7] that (3.1) also has a positive solution, which is a contradiction. Hence, $\left\{y_{n}\right\}$ belongs to class (II), and the first two inequalities in class (II) imply Property (B) of (1.1). This completes the proof.

Applying known oscillation criteria to (3.1), we immediately obtain criteria for Property (B) of (1.1), see for example [9], [14].

Corollary 3.2. Let $\left\{z_{n}\right\}$ be a positive solution of (2.1) satisfying (2.4) and (2.5). Let $f(u)=u$. If

$$
\liminf _{n \rightarrow \infty} \sum_{s=n-\ell}^{n-1} q_{s} z_{s+1} \sum_{t=n_{1}}^{s-\ell-1} z_{t} \sum_{j=n_{1}}^{t-1} \frac{1}{z_{j} z_{j+1}}>\left(\frac{\ell}{\ell+1}\right)^{\ell+1},
$$

then (1.1) has Property (B). 
Corollary 3.3. Let $\left\{z_{n}\right\}$ be a positive solution of (2.1) satisfying (2.4) and (2.5). Let $f(u)=u^{\alpha}$, where $\alpha \in(0,1)$ is a quotient of odd positive integers. If

$$
\sum_{n=n_{1}}^{\infty} q_{n} z_{n+1}\left(\sum_{s=n_{1}}^{n-\ell-1} z_{s} \sum_{t=n_{1}}^{s-1} \frac{1}{z_{t} z_{t+1}}\right)^{\alpha}=\infty
$$

then (1.1) has Property (B).

Corollary 3.4. Let $\left\{z_{n}\right\}$ be a positive solution of (2.1) satisfying (2.4) and (2.5). Let $f(u)=u^{\alpha}$, where $\alpha>1$ is a quotient of odd positive integers. If there exists $\lambda>\frac{\log \alpha}{\ell}$ such that

$$
\liminf _{n \rightarrow \infty} q_{n} z_{n+1}\left(\sum_{s=n_{1}}^{n-\ell-1} z_{s} \sum_{t=n_{1}}^{s-1} \frac{1}{z_{t} z_{t+1}}\right)^{\alpha} \exp \left(-e^{\lambda n}\right)>0,
$$

then (1.1) has Property (B).

Next, we are interested in finding criteria for the oscillation of all solutions of (1.1). Note that, by Theorem 3.1, if (3.1) is oscillatory, then (1.1) has Property (B). Therefore, it follows from Lemma 2.5 that by eliminating the possibility for nonoscillatory solutions belonging to class (II), one obtains sufficient conditions for the oscillation of all solutions of (1.1).

Theorem 3.5. Let $\left\{z_{n}\right\}$ be a positive solution of (2.1) such that (2.4) and (2.5) hold. If there exists $k \in \mathbb{N}$ with $\ell>2 k$ such that both (3.1) and

$$
\Delta x_{n}+z_{n} f\left(x_{n-\ell+2 k}\right) \sum_{s=n}^{n+k} \frac{1}{z_{s} z_{s+1}} \sum_{t=s}^{s+k} q_{t} z_{t+1}=0
$$

are oscillatory, then every solution of (1.1) is oscillatory.

Proof. Let $\left\{y_{n}\right\}$ be an eventually positive solution of (1.1). Then, by Lemma 2.5, $\left\{y_{n}\right\}$ belongs to either class (I) or (II). From Theorem 3.1, oscillation of (3.1) excludes the possibility of nonoscillatory solutions belonging to class (I). Therefore, $\left\{y_{n}\right\}$ belongs to class (II). Summation of (2.3) from $n$ to $n+k$ yields

$$
z_{n} z_{n+1} \Delta\left(\frac{\Delta y_{n}}{z_{n}}\right) \geq \sum_{s=n}^{n+k} q_{s} z_{s+1} f\left(y_{s-\ell}\right) \geq f\left(y_{n-\ell+k}\right) \sum_{s=n}^{n+k} q_{s} z_{s+1},
$$

i.e.,

$$
\Delta\left(\frac{\Delta y_{n}}{z_{n}}\right) \geq \frac{f\left(y_{n-\ell+k}\right)}{z_{n} z_{n+1}} \sum_{s=n}^{n+k} q_{s} z_{s+1}
$$


Summing (3.8) from $n$ to $n+k$, we obtain

$$
-\frac{\Delta y_{n}}{z_{n}} \geq f\left(y_{n-\ell+2 k}\right) \sum_{s=n}^{n+k} \frac{1}{z_{s} z_{s+1}} \sum_{t=s}^{s+k} q_{t} z_{t+1} .
$$

Again summing the last inequality from $n$ to $\infty$ yields

$$
y_{n} \geq \sum_{u=n}^{\infty} z_{u} f\left(y_{u-\ell+2 k}\right) \sum_{s=u}^{u+k} \frac{1}{z_{s} z_{s+1}} \sum_{t=s}^{s+k} q_{t} z_{t+1} .
$$

Let us denote by $x_{n}$ the right-hand side of (3.9). Clearly, $y_{n} \geq x_{n}>0$, and one can easily see that $\left\{x_{n}\right\}$ is a positive solution of the delay difference inequality

$$
\Delta x_{n}+z_{n} f\left(x_{n-\ell+2 k}\right) \sum_{s=n}^{n+k} \frac{1}{z_{s} z_{s+1}} \sum_{t=s}^{s+k} q_{t} z_{t+1} \leq 0 .
$$

But by [17, Lemma 2.7], (3.7) also has a positive solution, which is a contradiction. Thus, we conclude that every solution of (1.1) is oscillatory. This completes the proof.

Again applying known oscillation criteria to both (3.1) and (3.7), we obtain explicit conditions that ensure oscillation of all solutions of (1.1), see for example [9], [14].

Corollary 3.6. Let $\left\{z_{n}\right\}$ be a positive solution of (2.1) such that (2.4) and (2.5) hold. Let $f(u)=u$ and assume there exists $k \in \mathbb{N}$ with $\ell>2 k$. If (3.4) and

$$
\liminf _{n \rightarrow \infty} \sum_{s=n-\ell+2 k}^{n-1} z_{s} \sum_{t=s}^{s+k} \frac{1}{z_{t} z_{t+1}} \sum_{j=t}^{t+k} q_{j} z_{j+1}>\left(\frac{\ell-2 k}{\ell-2 k+1}\right)^{\ell-2 k+1}
$$

hold, then every solution of (1.1) is oscillatory.

Corollary 3.7. Let $\left\{z_{n}\right\}$ be a positive solution of (2.1) such that (2.4) and (2.5) hold. Let $f(u)=u^{\alpha}$, where $\alpha \in(0,1)$ is a ratio of odd positive integers. Assume that there exists $k \in \mathbb{N}$ with $\ell>2 k$. If (3.5) and

$$
\sum_{n=n_{1}}^{\infty} z_{n} \sum_{s=n}^{n+k} \frac{1}{z_{s} z_{s+1}} \sum_{t=s}^{s+k} q_{t} z_{t+1}=\infty
$$

hold, then every solution of (1.1) is oscillatory.

Corollary 3.8. Let $\left\{z_{n}\right\}$ be a positive solution of (2.1) such that (2.4) and (2.5) hold. Let $f(u)=u^{\alpha}$, where $\alpha>1$ is a ratio of odd positive integers. 
Assume that there exists $k \in \mathbb{N}$ such that $\ell>2 k$. If (3.6) holds and if there exists $\mu>\frac{\log \alpha}{\ell-2 k}$ such that

$$
\liminf _{n \rightarrow \infty} z_{n} \exp \left(-e^{\mu n}\right) \sum_{s=n}^{n+k} \frac{1}{z_{s} z_{s+1}} \sum_{t=s}^{s+k} q_{t} z_{t+1}>0,
$$

then every solution of (1.1) is oscillatory.

4. Examples. In this section, we present some examples in order to illustrate the main results.

Example 4.1. Consider the third-order delay difference equation

$$
\Delta^{3} y_{n}-\frac{2}{n(n+2)} \Delta y_{n+1}+\frac{3}{n+1} y_{n-2}=0, \quad n \in \mathbb{N} .
$$

Here,

$$
p_{n}=\frac{2}{n(n+2)}, \quad q_{n}=\frac{3}{n+1}, \quad \ell=2 .
$$

Now, (2.1) takes the form

$$
\Delta^{2} z_{n}-\frac{2}{n(n+2)} z_{n+1}=0, \quad n \in \mathbb{N} .
$$

Since $\left\{p_{n}\right\}$ is positive, (4.2) is nonoscillatory and has a positive solution $\left\{z_{n}\right\}=\{1 / n\}$, which satisfies (2.4) and (2.5). Also,

$$
\begin{aligned}
\liminf _{n \rightarrow \infty} \sum_{s=n-2}^{n-1} \frac{3}{(s+1)^{2}} \sum_{t=1}^{s-3} \frac{1}{t} & \sum_{j=1}^{t-1} j(j+1) \\
& =\liminf _{n \rightarrow \infty} \sum_{s=n-2}^{n-1} \frac{(s-3)(s-4)(2 s-1)}{6(s+1)^{2}}=\infty .
\end{aligned}
$$

Hence, (3.4) is satisfied. Therefore, by Corollary 3.2, (4.1) has Property (B).

Example 4.2. Consider the third-order delay difference equation

$$
\Delta^{3} y_{n}-\frac{2}{n(n+2)^{2}} \Delta y_{n+1}+(n+1) y_{n-3}^{1 / 3}=0, \quad n \in \mathbb{N} .
$$

Here,

$$
p_{n}=\frac{2}{n(n+2)^{2}}, \quad q_{n}=n+1, \quad \ell=3, \quad f(u)=u^{\alpha}, \quad \alpha=\frac{1}{3} .
$$

Now, (2.1) takes the form

$$
\Delta^{2} z_{n}-\frac{2}{n(n+2)^{2}} z_{n+1}=0, \quad n \in \mathbb{N} .
$$

By Lemma 2.1, (4.4) is nonoscillatory and has a positive solution $\left\{z_{n}\right\}=$ $\left\{\frac{n+1}{n}\right\}$, which satisfies (2.4) and (2.5). By taking $k=1$, we see that $\ell>2 k$ 
and (3.5) and (3.11) are satisfied. Hence, by Corollary 3.7, every solution of (4.3) is oscillatory.

Example 4.3. Consider the third-order delay difference equation

$$
\Delta^{3} y_{n}-\frac{2}{n(n+2)^{2}} \Delta y_{n+1}+\left(8+\frac{4}{n(n+2)^{2}}\right) y_{n-4}=0, \quad n \in \mathbb{N} .
$$

Here,

$$
p_{n}=\frac{2}{n(n+2)^{2}}, \quad q_{n}=8+\frac{4}{n(n+2)^{2}}, \quad \ell=4, \quad f(u)=u .
$$

Now, (2.1) takes the form (4.4), and it has a positive solution $\left\{z_{n}\right\}=\left\{\frac{n+1}{n}\right\}$, which satisfies (2.4) and (2.5). By taking $k=1$, we see that $\ell>2 k$ and (3.4) and (3.10) are satisfied. Hence, by Corollary 3.6, every solution of (4.5) is oscillatory. In fact, $\left\{y_{n}\right\}=\left\{(-1)^{n}\right\}$ is one such solution of (4.5).

We conclude this paper with the following remarks.

Remark 4.4. In this paper, we study the asymptotic and oscillatory behavior of solutions of third-order delay difference equations with negative damping term. We transform (1.1) into an equation without damping term and then obtain conditions for the oscillation of (1.1). We stress that, contrary to many known results in the literature leading to the conclusion that all solutions are either oscillatory or tend to zero as $n \rightarrow \infty$, our results guarantee oscillation of all solutions of (1.1).

Remark 4.5. Further note that, in all results, the explicit form of one positive solution of (2.1) is needed. However, it is known that it is very difficult to find the explicit form of solutions to a second-order difference equation with variable coefficient. So it is interesting to obtain criteria for the oscillation of (1.1) without involving the solution of (2.1).

\section{REFERENCES}

[1] Agarwal, R. P., Difference Equations and Inequalities. Theory, Methods, and Applications, Marcel Dekker, Inc., New York, 2000.

[2] Agarwal, R. P., Bohner, M., Grace, S. R., O'Regan, D., Discrete Oscillation Theory, Hindawi Publishing Corporation, New York, 2005.

[3] Agarwal R. P., Grace, S. R., Oscillation of certain third-order difference equations, Comput. Math. Appl. 42 (3-5) (2001), 379-384,

[4] Agarwal, R. P., Grace, S. R., O'Regan, D., On the oscillation of certain third-order difference equations, Adv. Difference Equ. 3 (2005), 345-367.

[5] Aktaş, M. F., Tiryaki, A., Zafer, A., Oscillation of third-order nonlinear delay difference equations, Turkish J. Math. 36 (3) (2012), 422-436.

[6] Bohner, M., Dharuman, C., Srinivasan, R., Thandapani, E., Oscillation criteria for third-order nonlinear functional difference equations with damping, Appl. Math. Inf. Sci. 11 (3) (2017), 669-676.

[7] Grace, S. R., Agarwal, R. P., Graef J. R., Oscillation criteria for certain third order nonlinear difference equations, Appl. Anal. Discrete Math. 3 (1) (2009), 27-38. 
[8] Graef, J. R., Thandapani, E., Oscillatory and asymptotic behavior of solutions of third order delay difference equations, Funkcial. Ekvac. 42 (3) (1999), 355-369.

[9] Győri, I., Ladas, G., Oscillation Theory of Delay Differential Equations. With Applications, The Clarendon Press, Oxford University Press, New York, 1991,

[10] Parhi, N., Panda, A., Oscillatory and nonoscillatory behaviour of solutions of difference equations of the third order, Math. Bohem. 133 (1) (2008), 99-112.

[11] Saker, S. H., Alzabut, J. O., Mukheimer, A., On the oscillatory behavior for a certain class of third order nonlinear delay difference equations, Electron. J. Qual. Theory Differ. Equ. 67 (2010), 16 pp.

[12] Smith, B., Oscillation and nonoscillation theorems for third order quasi-adjoint difference equations, Portugal. Math. 45 (3) (1988), 229-243.

[13] Smith, B., Taylor, Jr., W. E., Nonlinear third-order difference equations: oscillatory and asymptotic behavior, Tamkang J. Math. 19 (3) (1988), 91-95.

[14] Tang, X., Liu, Y., Oscillation for nonlinear delay difference equations, Tamkang J. Math. 32 (4) (2001), 275-280.

[15] Thandapani, E., Mahalingam, K., Oscillatory properties of third order neutral delay difference equations, Demonstratio Math. 35 (2) (2002), 325-337.

[16] Thandapani, E., Pandian, S., Balasubramaniam, R. K., Oscillatory behavior of solutions of third order quasilinear delay difference equations, Stud. Univ. Žilina Math. Ser. 19 (1) (2005), 65-78.

[17] Thandapani, E., Selvarangam, S., Oscillation theorems for second order quasilinear neutral difference equations, J. Math. Comput. Sci. 2 (4) (2012), 866-879.

Martin Bohner

Missouri S\&T, Rolla

MO 65409-0020

USA

e-mail: bohner@mst .edu

Srinivasan Selvarangam

Presidency College

Department of Mathematics

Chennai 600005

India

e-mail: selvarangam.9962@gmail.com
Srinivasan Geetha

Presidency College

Department of Mathematics

Chennai 600005

India

e-mail: srigeethamano@gmail.com

Ethiraju Thandapani

University of Madras

Ramanujan Institute for Advanced Study

in Mathematics

Chennai 600005

India

e-mail: ethandapani@yahoo.co.in

Received February 21, 2018 